\title{
Rest optical spectroscopy of FeLoBALs and LoBALs at intermediate redshift
}

\section{Kentaro Aoki}

Subaru Telescope, National Astronomical Observatory of Japan

E-mail: kaoki@subaru.naoj.org

NLS1s and low-ionization BAL (LoBAL) QSOs appear to share observational properties such as weak [O III] and strong Fe II emission line. We have observed $\mathrm{H} \beta-[\mathrm{O}$ III] region of 23 iron LoBALs and LoBALs at intermediate redshift $(1.4<\mathrm{z}<2.6)$. Most of our sample are weak [O III] emitters, however, there is a large variety of the strength of optical Fe II.

Narrow-Line Seyfert 1 Galaxies and their place in the Universe - NLS1,

April 04-06, 2011

Milan Italy 


\section{Similarity of LoBALs and NLS1s}

Similarities between NLS1s and Low-ionization Broad Absorption Line Quasars (LoBAL) have been pointed out by [6]. LoBALs are quasars which show absorption troughs from not only high-ionization ions such as $\mathrm{C}$ IV, N V, Si IV but also Mg II, Al III, and Al II. Both NLS1s and LoBALs have low [O III] equivalent width, and large Fe II (optical)/H $\beta$ [5, 17]. Those characters are recently confirmed by [18] using a lager sample of LoBALs.

\section{FeLoBAL}

A fraction of LoBALs shows absorptions from excited fine-structure levels of the ground term and excited terms of Fe II and Fe III [11, 3, 4, 10]. They are called iron LoBALs (FeLoBALs). FeLoBALs are rare quasars, however, the Sloan Digital Sky Survey (SDSS; [16]) are dramatically increasing numbers of FeLoBALs. Many of them have a reddened continuum (see [10]). They are dusty and possible young and high Eddington ratio objects.

It is expected that FeLoBALs and LoBALs may share the similar spectral properties of weak [O III] and strong Fe II, however near-infrared spectroscopies aiming $\mathrm{H} \beta$, Fe II of intermediate redshift FeLoBALs are scarce. [9] observed two FeLoBALs, and [17] included one FeLoBAL at $z \sim 2$ in their sample. Near-infrared spectroscopy of a radio-loud FeLoBAL SDSS J155633.77+151757.3 has been published by [14]. Thus we started near-infrared spectroscopic observations of LoBALs and FeLoBALs at $z \sim 2$.

\section{Observations}

Most of targets are chosen from SDSS. Significant fraction of the sample are selected from [10] and searched in SDSS database by ourselves. The observed quasars are listed in Table 1. Their optical, i.e. rest-UV spectra in SDSS are shown in Figs. 1-3 except for Hawaii 167 and PSS J0052+2405 which have no SDSS spectra. Please refer to [8] and [7] for optical spectra of Hawaii 167 and PSS J0052+2405, respectively. The results of SDSS J0839+3805 and SDSS J1723+5553 have been published in [1] and [2], respectively.

We used the Cooled Infrared Spectrograph and Camera for OHS (CISCO: [13]) and the MultiObject Infrared Camera and Spectrograph (MOIRCS: [12]) attached to the Subaru 8.2-m telescope and SpeX [15] with IRTF 3.0-m telescope. The spectral resolutions were 300-400 and 750 by Subaru and IRTF, respectively.

\section{Preliminary Results}

Our near-infrared spectra are shown in Figs. 4-6. Most of our sample are weak [O III] emitters. SDSS 1019+0225 has, however, exceptionally strong [O III] emission line. Its rest [O III] $\lambda 5007$ equivalent width is $42 \AA$. There is a large variety of the strength of optical Fe II. Some FeLoBALs, for example, SDSS J0342+0045, SDSS 0834+5112, SDSS J0810+4806, SDSS 0943+5417, are very strong optical Fe II emitters, while SDSS J1028+5115 and SDSS J1547+4443 have weak optical Fe II emission line. We can easily notice that strong [O III] emitters such as SDSS J1333+4556, SDSS J0018+0015, SDSS J1723+5553 and SDSS 1019+0225 are weak optical Fe II emitter. All 

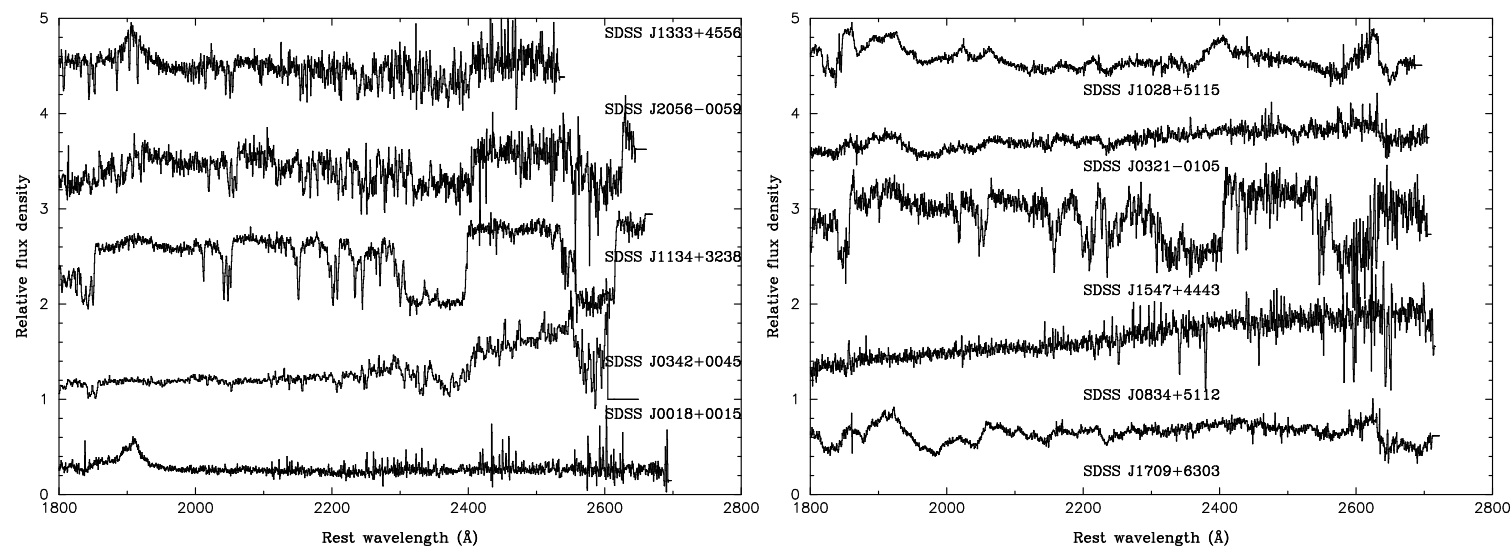

Figure 1: Rest UV spectra of the targets. The ordinate is the relative flux density $\left(f_{\lambda}\right)$, and the abscissa is the rest wavelength. Each spectrum is shifted in arbitrary step to avoid overlap. Higher redshift quasar is plotted upper.
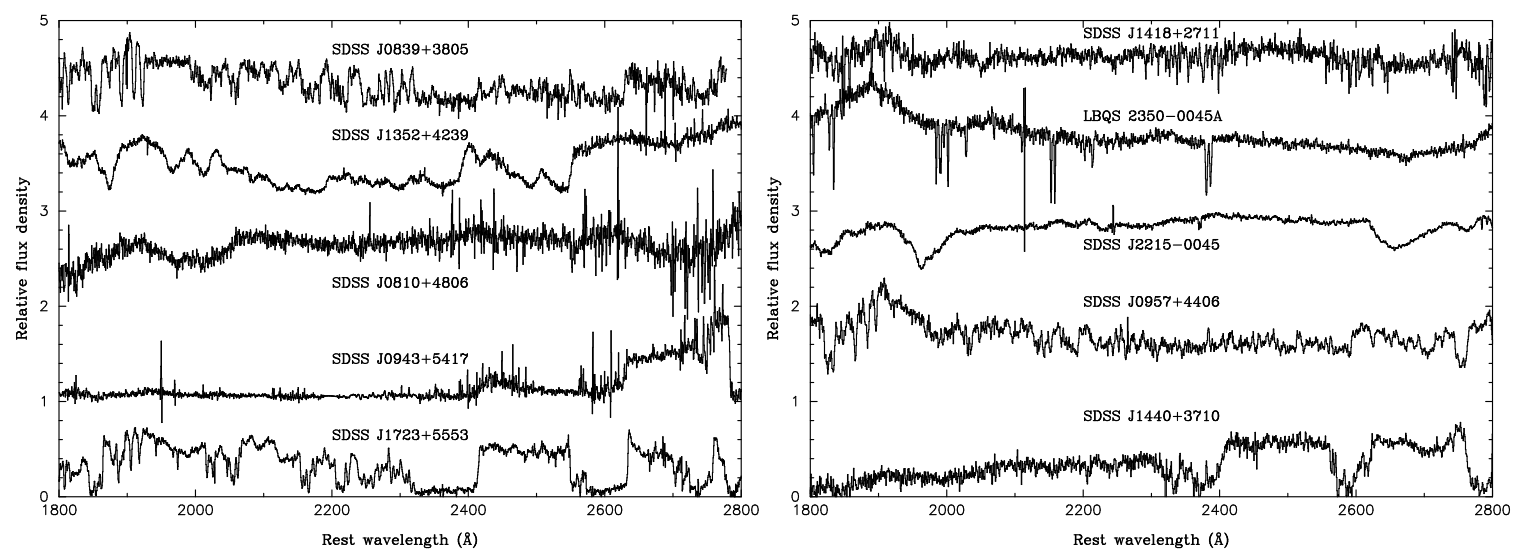

Figure 2: Rest UV spectra of the targets. Same as Fig.1.

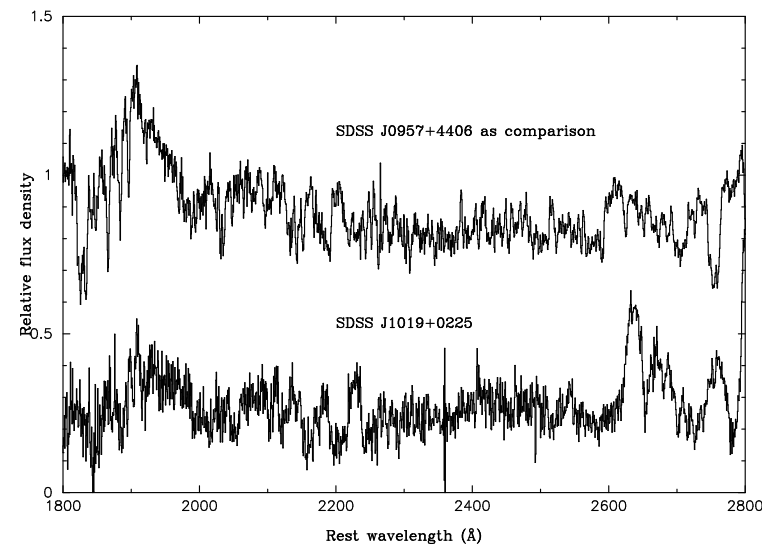

Figure 3: Rest UV spectra of the targets. Same as Fig.1, but for SDSS J1019+0225. SDSS 0957+4406 is shown again as comparison. 


\begin{tabular}{cccc}
\hline Name & Redshift & Telescope/Instrument & Obs. date (UT) \\
\hline SDSS J1019+0225 & 1.364 & IRTF/SpeX & 2007 Mar. 8 \\
SDSS J1440+3710 & 1.403 & IRTF/SpeX & 2007 Mar. 8 \\
SDSS J0957+4406 & 1.463 & IRTF/SpeX & 2007 Mar. 7 \\
SDSS J2215-0045 & 1.486 & Subaru/CISCO & 2004 Aug. 28 \\
LBQS 2350-0045A & 1.639 & Subaru/CISCO & 2004 Aug. 28 \\
SDSS J1418+2711 & 2.021 & Subaru/MOIRCS & 2011 Mar. 18 \\
SDSS J1723+5553 & 2.108 & Subaru/CISCO & 2005 Jul. 19 \\
SDSS J0943+5417 & 2.232 & Subaru/MOIRCS & 2011 Mar. 18 \\
SDSS J0810+4806 & 2.248 & Subaru/CISCO & 2003 Nov. 6 \\
SDSS J1352+4239 & 2.266 & IRTF/SpeX & 2007 Mar. 7 \\
SDSS J0839+3805 & 2.318 & Subaru/CISCO & 2005 Mar. 26 \\
Hawaii 167 & 2.347 & Subaru/CISCO & 2008 Mar. 20 \\
SDSS J1709+6303 & 2.386 & Subaru/CISCO & 2005 Jul. 26 \\
SDSS J0834+5112 & 2.395 & Subaru/CISCO & 2003 Nov. 6 \\
SDSS J1547+4443 & 2.403 & Subaru/CISCO & 2005 Apr. 1 \\
SDSS J0321-0105 & 2.406 & Subaru/CISCO & 2003 Oct. 18 \\
SDSS J1028+5110 & 2.420 & IRTF/SpeX & 2007 Mar. 9 \\
SDSS J0018+0045 & 2.428 & Subaru/CISCO & 2004 Jul. 11 \\
SDSS J0342+0045 & 2.435 & Subaru/CISCO & 2003 Oct. 17 \\
SDSS J1134+3238 & 2.454 & IRTF/SpeX & 2008 Mar. 7 \\
SDSS J2056-0059 & 2.476 & Subaru/MOIRCS & 2009 June 2 \\
PSS J0052+2405 & 2.500 & Subaru/CISCO & 2004 Jul. 11 \\
SDSS J1333+4556 & 2.629 & Subaru/MOIRCS & 2011 Mar. 18 \\
\hline & & &
\end{tabular}

Table 1: The targets' list.
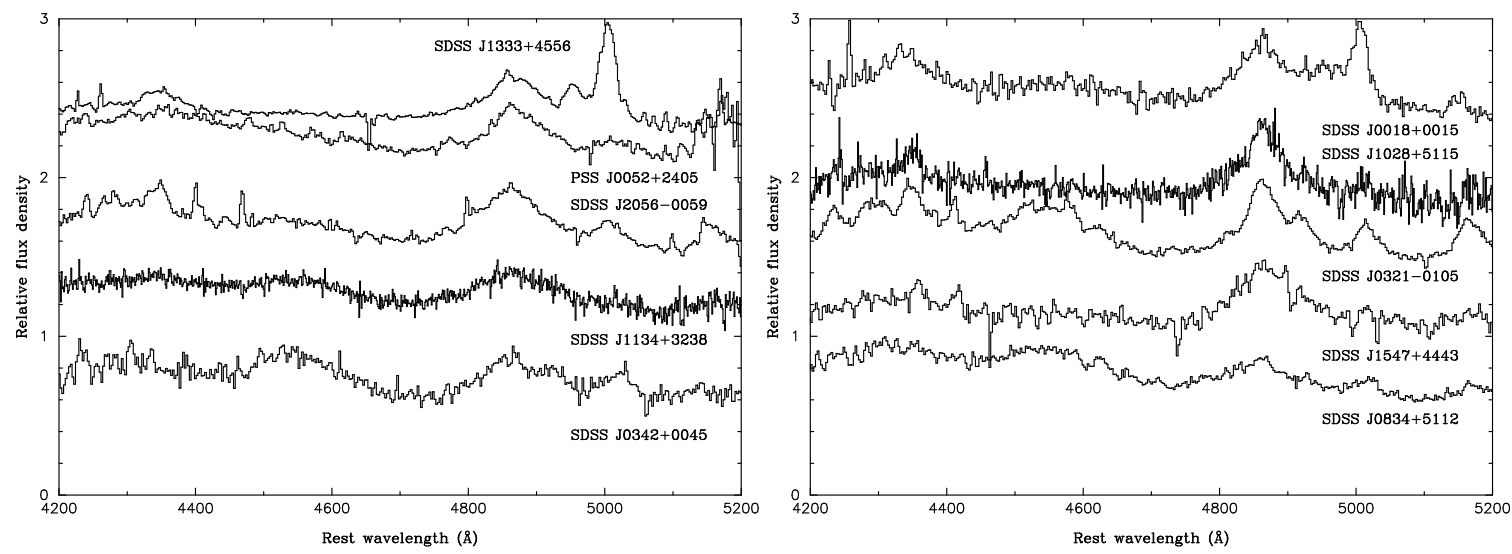

Figure 4: Rest optical spectra obtained by near-infrared spectroscopies. The ordinate is the relative flux density $\left(f_{\lambda}\right)$, and the abscissa is the rest wavelength. Each spectrum is shifted in arbitrary step to avoid overlap. Higher redshift quasar is plotted upper 

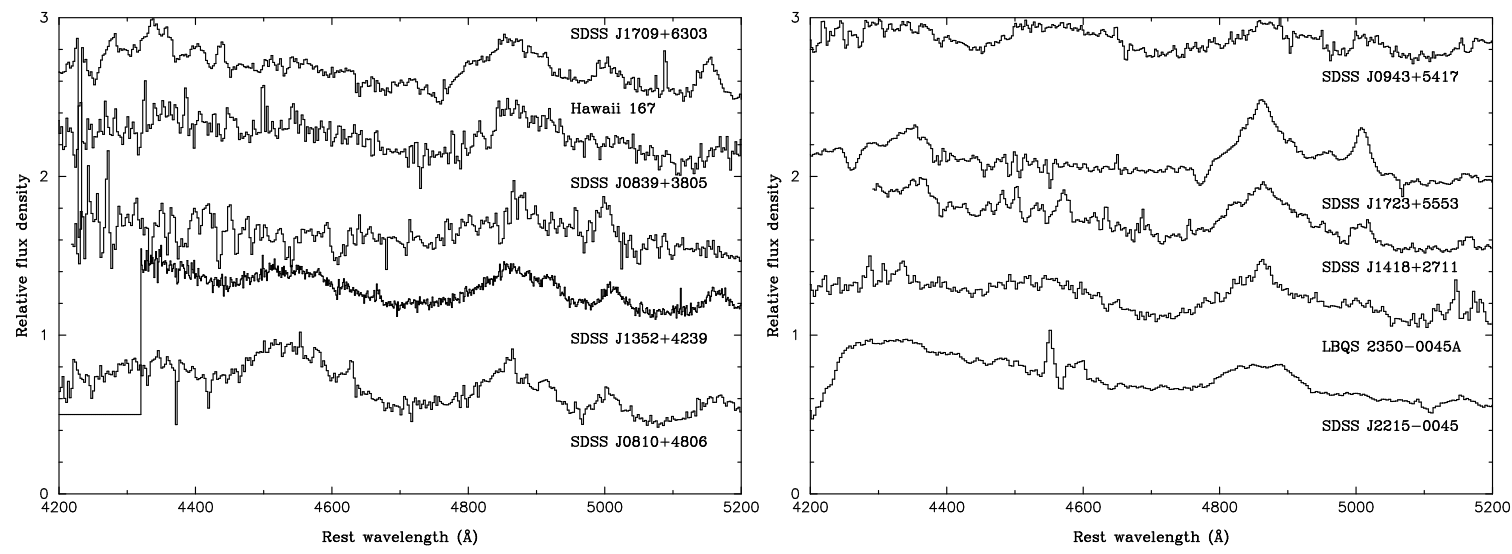

Figure 5: Rest optical spectra. Same as Fig. 4.

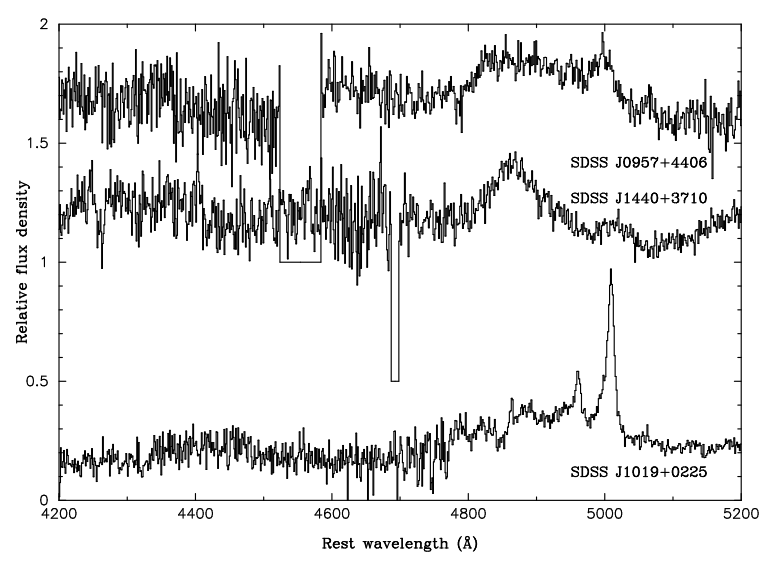

Figure 6: Rest optical spectra. Same as Fig. 4.

four objects except for SDSS 1019+0225 are radio-quiet. The anti-correlation between [O III] and optical Fe II strength seems to exist among our sample. The two FeLoBALs, SDSS J1028+5115 and SDSS J1547+4443 have weak [O III] and optical Fe II emission line, thus they might be outliers. FeLoBALs have wide variety of properties in absorption lines, line width, velocity shift, line ratios. The relationships between UV and optical spectral properties will be investigated in future.

\section{References}

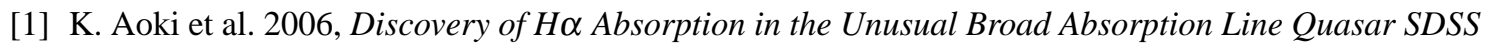
J083942.11+380526.3, ApJ, 651, 84

[2] K. Aoki 2010, Broad Balmer-Line Absorption in SDSS J172341.10+555340.5, PASJ, 62, 1333

[3] R. H. Becker et al. 1997, The First Radio-loud Broad Absorption Line QSO and Evidence for a Hidden Population of Quasars, ApJ, 479, L93

[4] R. H. Becker, et al. 2000, Properties of Radio-selected Broad Absorption Line Quasars from the First Bright Quasar Survey, ApJ, 538, 72

[5] T. A. Boroson \& K. A Meyers 1992, The optical properties of IR-selected and Mg II broad absorption line quasars, ApJ, 397, 442

[6] W. N. Brandt, \& S. C. Gallagher 2000, New Astronomy Reviews, 44, 461 
[7] R. J. Brunner, et al. 2003, Peculiar Broad Absorption Line Quasars Found in The Digitized Palomar Observatory Sky Survey, AJ, 126, 53

[8] L. L. Cowie, et al. 1994, Hawaii 167: A compact absorption-line object at Z = 2.35, ApJ, 432, L83

[9] E. Egami, et al. 1996, Hawaii 167 and Q0059-2735: Heavily Dust-Enshrouded Young QSOs, AJ, 112, 73

[10] P. B. Hall et al. 2002, Unusual Broad Absorption Line Quasars from the Sloan Digital Sky Survey, ApJS, 141, 267

[11] C. Hazard, et al. 1987, The remarkable broad absorption line QSO 0059-2735 with extensive Fe II absorption, ApJ, 323, 263

[12] T. Ichikawa, et al. 2006, MOIRCS: multi-object infrared camera and spectrograph for SUBARU, in proceedings of Society of Photo-Optical Instrumentation Engineers (SPIE) Conference Series, 6269, 38

[13] K. Motohara, et al. 2002, CISCO: Cooled Infrared Spectrograph and Camera for OHS on the Subaru Telescope, PASJ, 54, 315

[14] J. Najita, et al. 2000, Red Quasars and Quasar Evolution: The Case of BAL QSO FIRST $J 155633.8+351758, A J, \mathbf{1 2 0}, 2859$

[15] J. T. Rayner, et al. 2003, SpeX: A Medium-Resolution 0.8-5.5 Micron Spectrograph and Imager for the NASA Infrared Telescope Facility, PASP, 115, 362

[16] D. G. York, et al. 2000, The Sloan Digital Sky Survey: Technical Summary, AJ, 120, 1579

[17] M. J. Yuan, \& B. J. Wills 2003, Eddington Accretion and QSO Emission Lines at z 2, ApJ, 593, L11

[18] S. Zhang, et al. 2010, Low-z Mg II Broad Absorption-line Quasars from the Sloan Digital Sky Survey, ApJ, 714, 367 